\title{
Co-occurrence of spinal and intracranial tuberculoma in a child with juvenile rheumatoid arthritis
}

Gizem Timoçin, Huseyin Toprak, Serpil Kurtcan, Alpay Alkan

Department of Radiology, Medical Faculty, Bezmialem Vakif University, Istanbul, Turkey

Submitted: 24 January 2017

Accepted: 14 March 2017

Arch Med Sci Civil Dis 2017; 2: e55-e58

DOI: https://doi.org/10.5114/amscd.2017.66807

Copyright $\odot 2017$ Termedia \& Banach

Tuberculosis (TB) is one of the oldest known diseases, and TB is still an important pathological entity. The central nervous system (CNS) is involved in approximately $10 \%$ of all tuberculosis cases. Acquired immunodeficiency syndrome (AIDS) and immunosuppressive therapy such as steroids, anti-TNF (tumor necrosis factor) drugs and chemotherapeutic drugs have resulted in an increased incidence of TB worldwide [1]. It generally presents as an intracranial lesion, typically observed in the frontal and parietal lobes. Intramedullary spinal tuberculoma is very rare, occurring at a frequency of $0.2-5 \%$ [2]. It is typically observed in the dorsal column. Co-occurrence of intracerebral and intramedullary tuberculomas is extremely rare, with only a few cases reported in the literature [3]. The medications used for juvenile rheumatoid arthritis (JRA), including nonsteroidal anti-inflammatory drugs, disease-modifying antirheumatic drugs, TNF- $\alpha$ blockers and corticosteroids, can cause immunodeficiency. This immunosuppression can cause intracranial and spinal TB infection. Such a combination, to the best of our knowledge, has not been reported previously. For this reason, we report this rarely encountered manifestation in a child.

A 15-year-old girl presented with a 3-month history of fever and headache. The patient was diagnosed with juvenile rheumatoid arthritis (JRA) and use methotrexate (MTX $20 \mathrm{mg} /$ day) and methylprednisolone $(3 \times 16 \mathrm{mg})$. Hematological examination of the patient revealed normal parameters except increased $C$ reactive protein (4.06; reference $<0.5)$. Chest $X$-ray showed miliary deposits appearing as 1-3 mm diameter nodules, which were uniformly distributed (Figure 1$)$. After the chest $X$ ray examination miliary TB was diagnosed. Because of the symptoms in our case, we suspect CNS tuberculomas and cranio-spinal magnetic resonance imaging (MRI) was performed. Intracranial lesions located at the brainstem, cerebellum and dispersedly in both cerebral hemispheres were visualized. On T2 weighted images, lesions showed central hypointensity and perilesional hyperintense vasogenic edema. After contrast administration, nodular enhancement was noted (Figure 2).

\author{
Corresponding author: \\ Dr. Gizem Timoçin \\ Department of Radiology \\ Medical Faculty \\ Bezmialem Vakif University \\ Adnan Menderes Bulvarı \\ Vatan Caddesi \\ 34093 Fatih/Istanbul, Turkey \\ Phone: +902126610850 \\ E-mail: gizemtimocin1@ \\ gmail.com
}

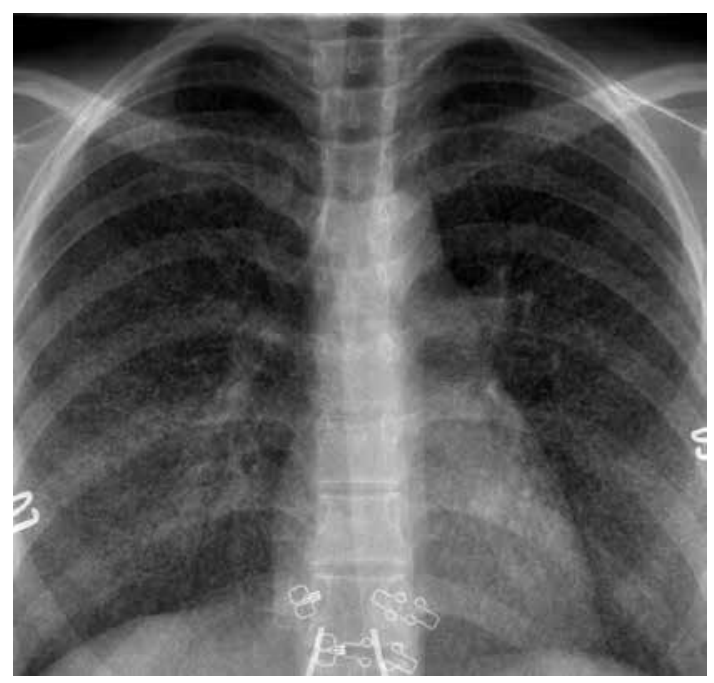

Figure 1. On chest $X$ ray (antero-posterior) examination miliary nodules are seen, which are $1-3 \mathrm{~mm}$ diameter and uniformly distributed 
Sagittal and axial T2-weighted spinal MRI showed intramedullary hypointense lesions at the thoracic T9 and T11 regions (Figure 3). T1 contrast images (fat saturated) showed nodular enhancement. In our case, pulmonary miliary TB was present and CNS tuberculosis probably originated from the lung by hematogenous dissemination. The MRI features suggested intracranial and spinal tuberculoma.

Following the diagnosis of tuberculosis, MTX therapy was stopped and anti-tuberculous che-
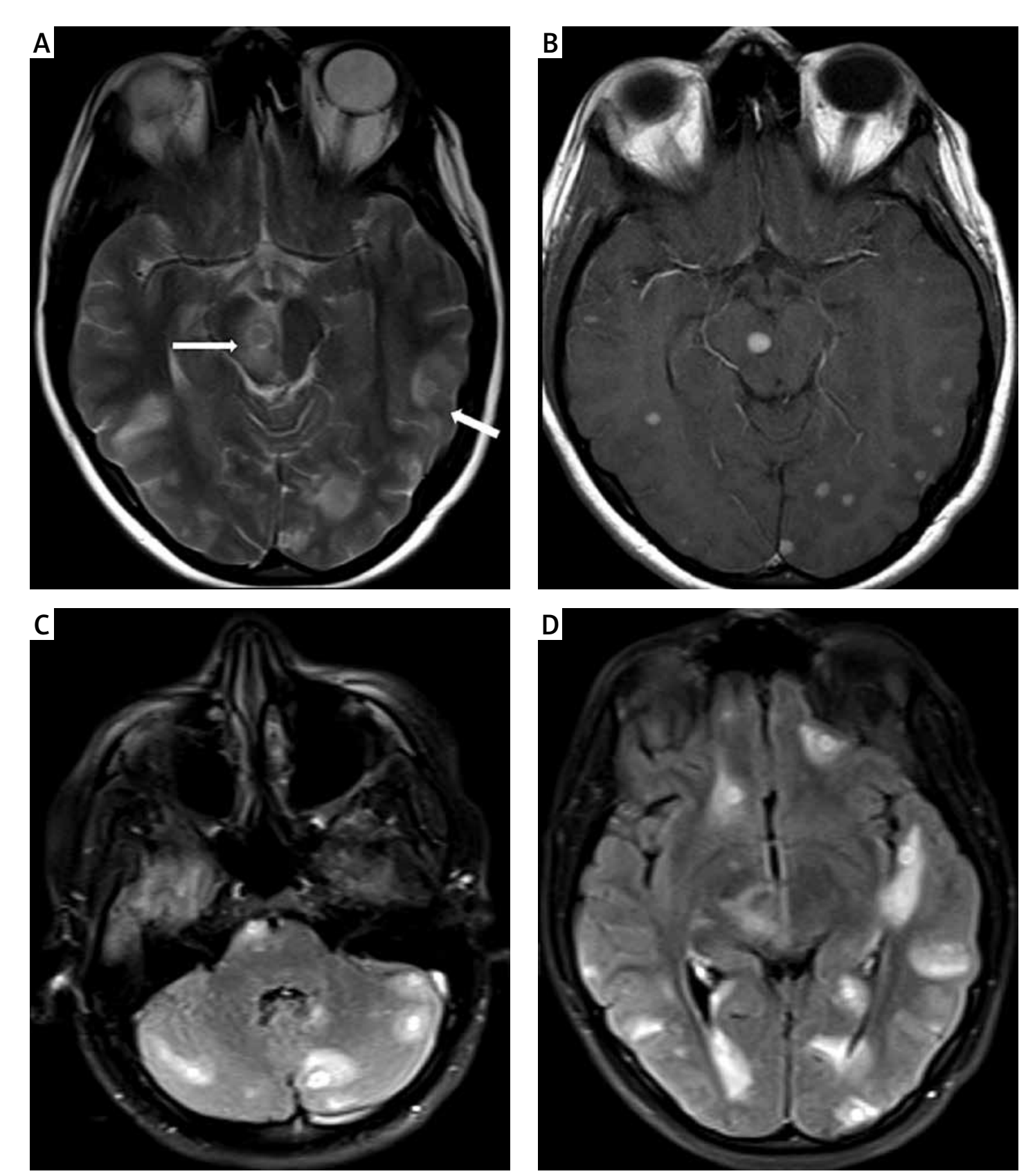

Figure 2. Axial plane cranial T2-weighted magnetic resonance imaging (MRI): A - central hypointensity and perilesional hyperintense vasogenic edema in both cerebral hemispheres, gadolinium-enhanced (fat saturated) axial T1-weighted MRI, B - nodular enhancement in mesencephalon and dispersedly in both cerebral hemispheres, axial plane flair MRI, C - central hypointensity and perilesional hyperintense vasogenic edema in cerebellum and brainstem, axial plane Flair MRI, D - central hypointensity and perilesional hyperintense vasogenic edema in both cerebral hemispheres motherapy was started (combination of isoniazid, rifampin, ethambutol and pyrazinamide).

The CNS is involved in approximately $10 \%$ of all tuberculosis cases. The most common manifestation of neurotuberculosis is tuberculous meningitis, and intracranial-parenchyma TB most commonly affects the frontal lobes. It commonly presents with fever, headache, intracranial hypertension, seizures, and focal neurological symptoms. The ratio of intramedullary TB to intracerebral TB is approximately $1 / 42$ [4], and spinal 

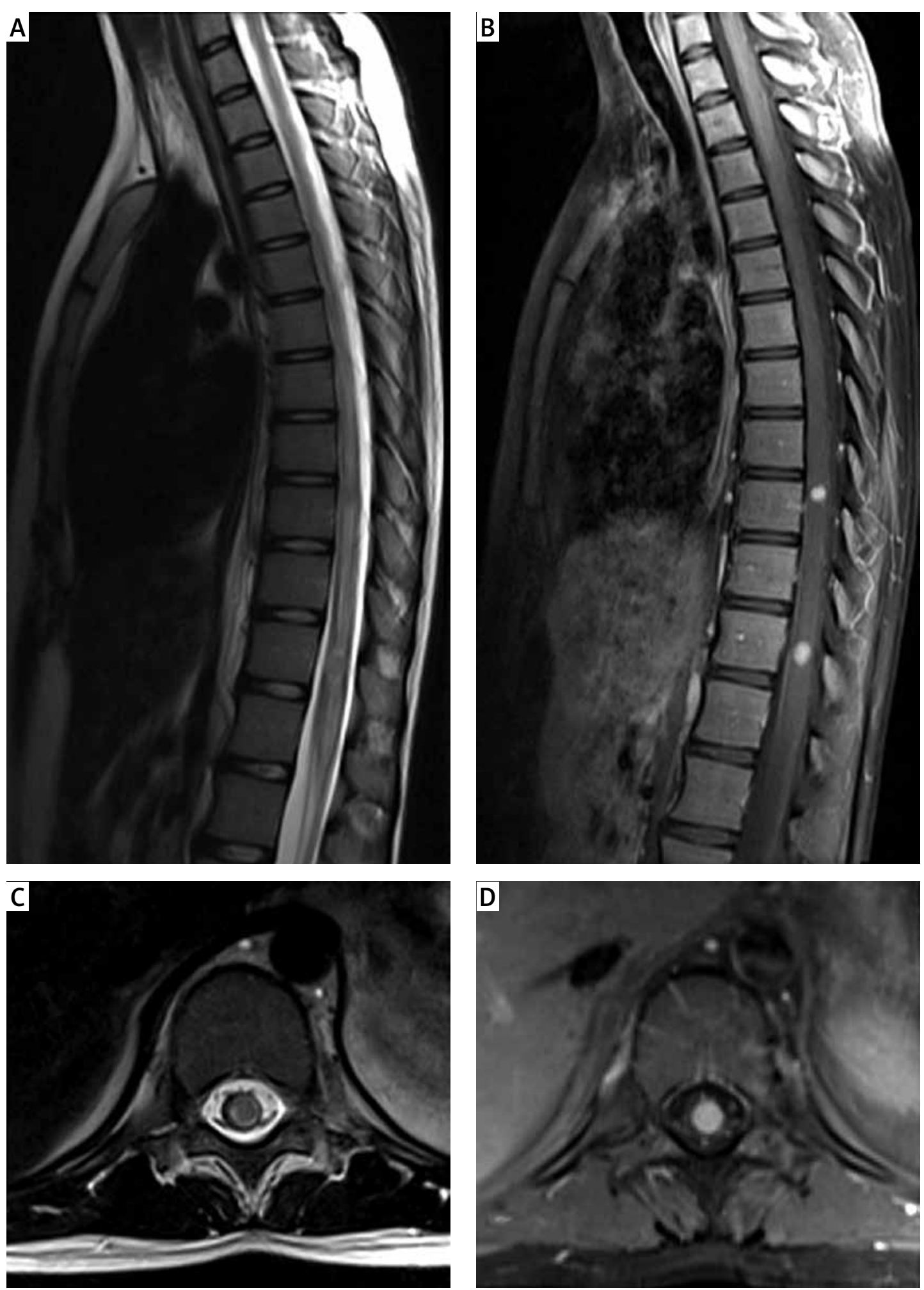

Figure 3. A - Sagittal T2-weighted magnetic magnetic resonance imaging MRI of the thoracic spine demonstrates intramedullary hypointense lesions at the thoracic T9 and T11 regions and perilesional vasogenic edema, B - gadolinium-enhanced (fat saturated) sagittal T1-weighted MRI shows nodular enhancement at the T9 and T11 levels, axial T2 weighted MRI, C - shows intramedullary hypointense lesion, D - corresponding nodular enhancing T1-contrast (fat saturated) axial image 
tuberculomas are commonly located in the thoracic cord [5]. Clinical symptoms and presentation of intramedullary spinal tuberculoma are usually indistinguishable from spinal cord tumors. Neurocysticercosis, cerebral toxoplasmosis, abscesses and metastasis are differential diagnoses of intracranial-spinal tuberculoma $[6,7]$.

Tuberculomas are isointense to grey matter and may have a central region of hyperintensity representing caseation on T1-weighted imaging. On T2-weighted imaging they are isointense to grey matter, may have a central region of hypointensity representing gliosis and abundant monocyte infiltration, and lesions are surrounded by vasogenic edema. Intramedullary tuberculomas also observed; they are T2 hypointense and surrounded by minimal vasogenic edema. In our case, tuberculomas (intracranial-intramedullary) had central hypointensity representing caseation, surrounded by vasogenic edema on T2-weighted imaging. On T1-weighted imaging tuberculomas were isointense to grey matter. In the literature tuberculomas typically showed peripheral enhancement, but in our case both intracranial and intramedullary tuberculomas showed nodular enhancement, not peripheral. The diagnosis is made on the basis of neuroimaging findings.

Co-occurrence of intracerebral and intramedullary tuberculomas is rare. Immunosuppressive therapy such as steroids, anti-TNF drugs, chemotherapeutic drugs and AIDS can cause intracranial and spinal tuberculoma in childhood. TNF- $\alpha$ has a central role in mycobacterial infection and disease. TNF blockers in particular have been associated with increased prevalence of TB. In our case, the patient uses MTX and MTX inhibits the production of intracellular polyamines, which are essential for lymphocyte growth and replication. Freidman et al. and many others have confirmed that MTX depresses established delayed hypersensitivity [8]. A study suggested that MTX promotes the apoptosis of activated T cells, which in turn limits $T$ cell proliferation. According to this hypothesis, the incidence of tuberculosis has increased depending on immunosuppression.

In the case of a child presenting with headache and neurological signs who has undergone immunosuppressive therapy for JRA, one should bear in mind the possibility of intracranial-intramedullary tuberculosis involvement. In the case of suspicion of CNS tuberculomas, contrast-enhanced MRI should be performed.

\section{Conflict of interest}

The authors declare no conflict of interest.

\section{References}

1. Trivedi R, Saksena S, Gupta RK. Magnetic resonance imaging in central nervous system tuberculosis. Indian J Radiol Imaging 2009; 19: 256-65.

2. Thacker MM, Puri Al. Concurrent intra-medullary and intra-cranial tuberculomas. J Postgrad Med 2004; 50: 107-9.

3. Vera R, Kumar S. Concurrent intramedullary and intracranial tuberculoma: a rare association. Ann Trop Med Public Health 2015; 8: 132-4

4. Citow JS, Ammirati M. Intramedullary tuberculoma of the spinal cord: case report. Neurosurgery 1994; 35: 327-30.

5. Gülsen S, Caner H. Tuberculoma in the medulla oblangata and medulla spinalis: two case reports. Balkan Med J 2013; 30: 442-5.

6. Tekin R, Kacar E, Cevik FC, Cinar K, Cevik R. Intracranial and intramedullary tuberculoma with intravertebral abscess manifestation under anti-tuberculous treatment: a case report. Clin Neurol Neurosurg 2013; 115: 185860.

7. Yen HL, Lee RJ, Lin JW, Chen HJ. Multiple tuberculomas in the brain and spinal cord: a case report. Spine 2003; 28: 499-502.

8. Friedman RM, Buckler CE. Mechanism of MTX action on tuberculin hypersensitivity inguinea-pigs. Fend $\operatorname{Pr} 1963$; 22: 501-5. 